\title{
On the Book Thickness of $k$-Trees
}

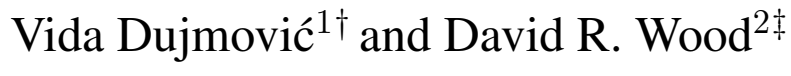 \\ ${ }^{1}$ School of Computer Science, Carleton University, Ottawa, Canada \\ ${ }^{2}$ Department of Mathematics and Statistics, The University of Melbourne, Melbourne, Australia
}

received $25^{\text {th }}$ November 2010, accepted $26^{\text {th }}$ September 2011.

Every $k$-tree has book thickness at most $k+1$, and this bound is best possible for all $k \geq 3$. Vandenbussche et al. [SIAM J. Discrete Math., 2009] proved that every $k$-tree that has a smooth degree-3 tree decomposition with width $k$ has book thickness at most $k$. We prove this result is best possible for $k \geq 4$, by constructing a $k$-tree with book thickness $k+1$ that has a smooth degree- 4 tree decomposition with width $k$. This solves an open problem of Vandenbussche et al.

MSC: 05C62, 68R10

Keywords: graph, book embedding, book thickness, pagenumber, stacknumber, treewidth, tree decomposition

\section{Introduction}

Consider a drawing of a graph ${ }^{(i)} G$ in which the vertices are represented by distinct points on a circle in the plane, and each edge is a chord of the circle between the corresponding points. Suppose that each edge is assigned one of $k$ colours such that crossing edges receive distinct colours. This structure is called a $k$ page book embedding of $G$ : one can also think of the vertices as being ordered along the spine of a book, and the edges that receive the same colour being drawn on a single page of the book without crossings. The book thickness of $G$, denoted by bt $(G)$, is the minimum integer $k$ for which there is a $k$-page book embedding of $G$. Book embeddings, first defined by Ollmann (1973), are ubiquitous structures with a variety of applications; see (Dujmović and Wood, 2004) for a survey with over 50 references. A book embedding is also called a stack layout, and book thickness is also called stacknumber, pagenumber and fixed outerthickness.

This paper focuses on the book thickness of $k$-trees. A vertex $v$ in a graph $G$ is $k$-simplicial if its neighbourhood, $N_{G}(v)$, is a $k$-clique. For $k \geq 1$, a $k$-tree is a graph $G$ such that either $G \simeq K_{k+1}$, or $G$ has a $k$-simplicial vertex $v$ and $G-v$ is a $k$-tree. In the latter case, we say that $G$ is obtained from $G-v$ by adding $v$ onto the $k$-clique $N_{G}(v)$.



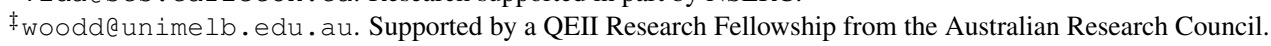

(i) We consider simple, finite, undirected graphs $G$ with vertex set $V(G)$ and edge set $E(G)$. We employ standard graph-theoretic terminology; see (Diestel 2000). For disjoint $A, B \subseteq V(G)$, let $G[A ; B]$ denote the bipartite subgraph of $G$ with vertex set $A \cup B$ and edge set $\{v w \in E(G): v \in A, w \in B\}$.

1365-8050 @ 2011 Discrete Mathematics and Theoretical Computer Science (DMTCS), Nancy, France 
What is the maximum book thickness of a $k$-tree? Observe that 1-trees are precisely the trees. Bernhart and Kainen (1979) proved that every 1-tree has a 1-page book embedding. In fact, a graph has a 1-page book embedding if and only if it is outerplanar (Bernhart and Kainen, 1979). 2-trees are the edge-maximal series-parallel graphs. Rengarajan and Veni Madhavan (1995) proved that every series parallel graph, and thus every 2-tree, has a 2-page book embedding (also see (Di Giacomo et al., 2006)). This bound is best possible, since $K_{2,3}$ is series parallel and is not outerplanar. Ganley and Heath (2001) proved that every $k$-tree has a $(k+1)$-page book embedding; see (Dujmović and Wood. 2007) for an alternative proof. Ganley and Heath (2001) also conjectured that every $k$-tree has a $k$-page book embedding. This conjecture was refuted by Dujmović and Wood (2007), who constructed a $k$-tree with book thickness $k+1$ for all $k \geq 3$. Vandenbussche et al. (2009) independently proved the same result. Therefore the maximum book thickness of a $k$-tree is $k$ for $k \leq 2$ and is $k+1$ for $k \geq 3$.

Which families of $k$-trees have $k$-page book embeddings? Togasaki and Yamazaki (2002) proved that every graph with pathwidth $k$ has a $k$-page book embedding (and there are graphs with pathwidth $k$ and book thickness $k$ ). This result is equivalent to saying that every $k$-tree that has a smooth degree- 2 tree decomposition (ii) of width $k$ has a $k$-page book embedding. Vandenbussche et al. (2009) extended this result by showing that every $k$-tree that has a smooth degree-3 tree decomposition of width $k$ has a $k$-page book embedding. Vandenbussche et al. (2009) then introduced the following natural definition. Let $m(k)$ be the maximum integer $d$ such that every $k$-tree that has a smooth degree- $d$ tree decomposition of width $k$ has a $k$-page book embedding. Vandenbussche et al. (2009) proved that $3 \leq m(k) \leq k+1$, and state that determining $m(k)$ is an open problem. However, it is easily seen that the $k$-tree with book thickness $k+1$ constructed in (Dujmović and Wood, 2007) has a smooth degree-5 tree decomposition with width $k$. Thus $m(k) \leq 4$ for all $k \geq 3$. The main result of this note is to refine the construction in (Dujmović and Wood 2007) to give a $k$-tree with book thickness $k+1$ that has a smooth degree-4 tree decomposition with width $k$ for all $k \geq 4$. This proves that $m(k)=3$ for all $k \geq 4$. It is open whether $m(3)=3$ or 4 . We conjecture that $m(3)=3$.

\section{Construction}

Theorem 1 For all $k \geq 4$ and $n \geq 11\left(2 k^{2}+1\right)+k$, there is an $n$-vertex $k$-tree $Q$, such that $\operatorname{bt}(Q)=k+1$ and $Q$ has a smooth degree-4 tree decomposition of width $k$.

Proof: Start with the complete split graph $K_{k, 2 k^{2}+1}^{\star}$. That is, $K_{k, 2 k^{2}+1}^{\star}$ is the $k$-tree obtained by adding a set $S$ of $2 k^{2}+1$ vertices onto a $k$-clique $K=\left\{u_{1}, u_{2}, \ldots, u_{k}\right\}$, as illustrated in Figure 1 For each vertex $v \in S$ add a vertex onto the $k$-clique $(K \cup\{v\}) \backslash\left\{u_{1}\right\}$. Let $T$ be the set of vertices added in this step. For each $w \in T$, if $v$ is the neighbour of $w$ in $S$, then add a set $T_{2}(w)$ of three simplicial vertices onto the $k$-clique $(K \cup\{v, w\}) \backslash\left\{u_{1}, u_{2}\right\}$, add a set $T_{3}(w)$ of three simplicial vertices onto the $k$-clique $(K \cup\{v, w\}) \backslash\left\{u_{1}, u_{3}\right\}$, and add a set $T_{4}(w)$ of three simplicial vertices onto the $k$-clique $(K \cup\{v, w\}) \backslash$ $\left\{u_{1}, u_{4}\right\}$. This step is well defined since $k \geq 4$. For each $w \in T$, let $T(w):=T_{2}(w) \cup T_{3}(w) \cup T_{4}(w)$. By construction, $Q$ is a $k$-tree, and as illustrated in Figure $2, Q$ has a smooth degree-4 tree decomposition of width $k$.

\footnotetext{
(ii) See (Diestel, 2000) for the definition of tree decomposition and treewidth. Note that $k$-trees are the edge maximal graphs with treewidth $k$. A tree decomposition of width $k$ is smooth if every bag has size exactly $k+1$ and any two adjacent bags have exactly $k$ vertices in common. Any tree decomposition of a graph $G$ can be converted into a smooth tree decomposition of $G$ with the same width. A tree decomposition is degree- $d$ if the host tree has maximum degree at most $d$.
} 


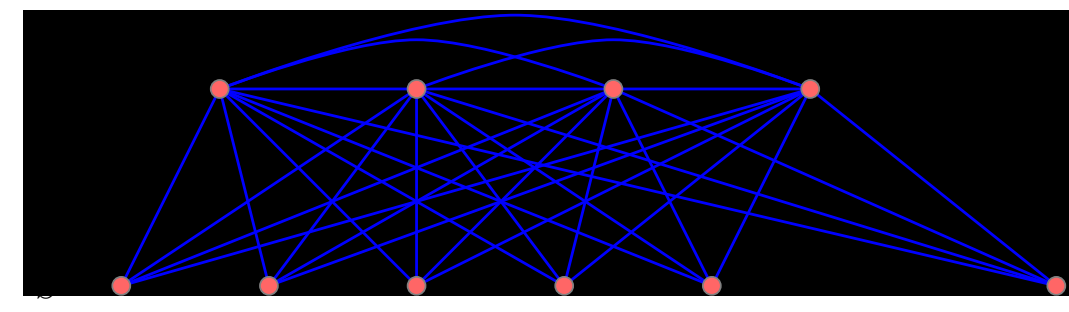

Fig. 1: The complete split graph $K_{4,|S|}^{\star}$.

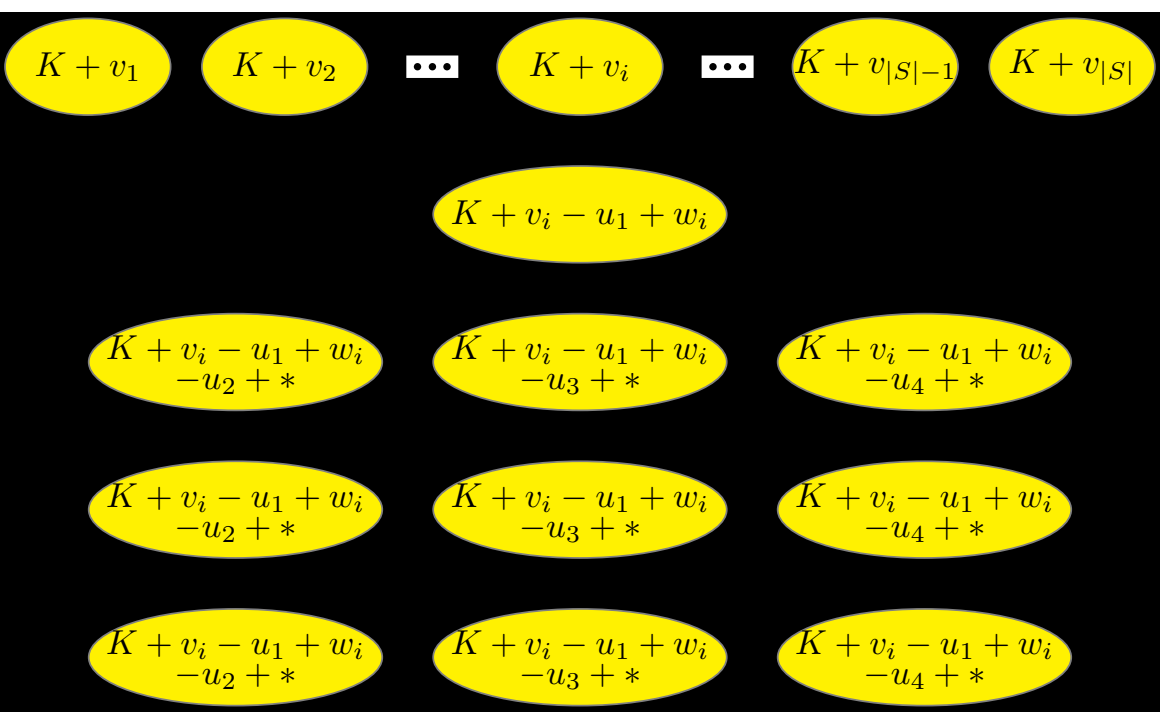

Fig. 2: A smooth degree-4 tree decomposition of $Q$.

It remains to prove that $\operatorname{bt}(Q) \geq k+1$. Suppose, for the sake of contradiction, that $Q$ has a $k$-page book embedding. Say the edge colours are $1,2, \ldots, k$. For each ordered pair of vertices $v, w \in V(Q)$, let $\widehat{v w}$ be the list of vertices in clockwise order from $v$ to $w$ (not including $v$ and $w$ ).

Say $K=\left(u_{1}, u_{2}, \ldots, u_{k}\right)$ in anticlockwise order. Since there are $2 k^{2}+1$ vertices in $S$, by the pigeonhole principle, without loss of generality, there are at least $2 k+1$ vertices in $S \cap \widehat{u_{1} u_{k}}$. Let $\left(v_{1}, v_{2}, \ldots, v_{2 k+1}\right)$ be $2 k+1$ vertices in $S \cap \widehat{u_{1} u_{k}}$ in clockwise order.

Observe that the $k$ edges $\left\{u_{i} v_{k-i+1}: 1 \leq i \leq k\right\}$ are pairwise crossing, and thus receive distinct colours, as illustrated in Figure 3 a). Without loss of generality, each $u_{i} v_{k-i+1}$ is coloured $i$. As illustrated in Figure 3 (b), this implies that $u_{1} v_{2 k+1}$ is coloured 1 , since $u_{1} v_{2 k+1}$ crosses all of $\left\{u_{i} v_{k-i+1}: 2 \leq i \leq\right.$ $k\}$ which are coloured $2,3, \ldots, k$. As illustrated in Figure $3(\mathrm{c})$, this in turn implies that $u_{2} v_{2 k}$ is coloured 2 , and so on. By an easy induction, $u_{i} v_{2 k+2-i}$ is coloured $i$ for each $i \in\{1,2, \ldots, k\}$, as illustrated in Figure 3 (d). It follows that for all $i \in\{1,2, \ldots, k\}$ and $j \in\{k-i+1, k-i+2, \ldots, 2 k+2-i\}$, the edge $u_{i} v_{j}$ is coloured $i$, as illustrated in Figure 3 (e). Moreover, as illustrated in Figure 3 (f): 
If $q u_{i} \in E(Q)$ and $q \in \widehat{v_{k} v_{k+2}}$, then $q u_{i}$ is coloured $i$.
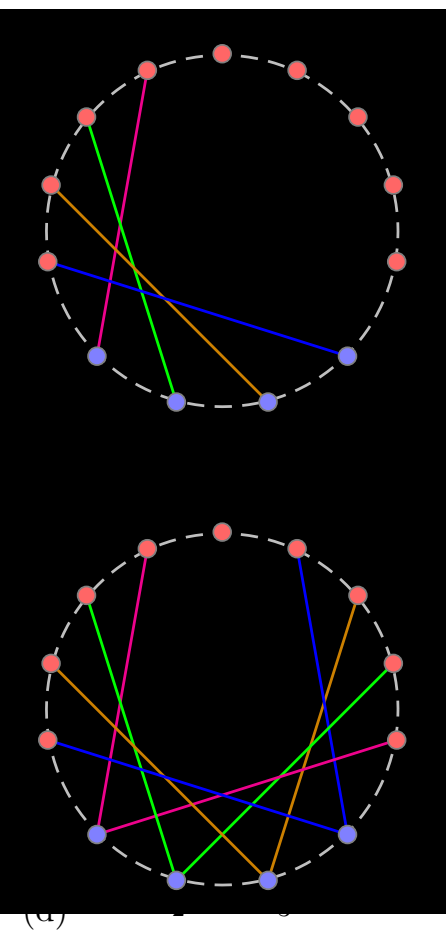

(b)
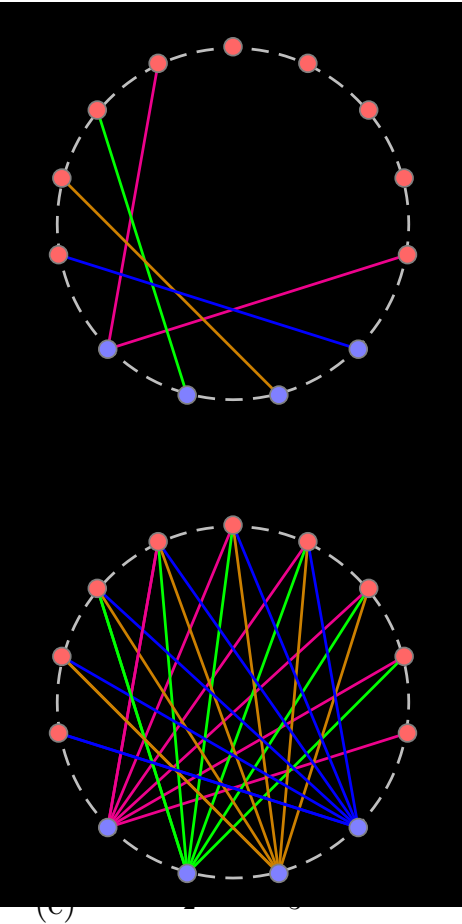



(c)



Fig. 3: Illustration of the proof of Theorem 1 with $k=4$.

Note that the argument up to now is the same as in (Dujmović and Wood, 2007). Let $w$ be the vertex in $T$ adjacent to $v_{k+1}$. Recall that $w$ is adjacent to each vertex in $K \backslash\left\{u_{1}\right\}$. Vertex $w$ is in $\widehat{v_{k} v_{k+2}}$, as otherwise the edge $w v_{k+1}$ crosses $k$ edges of $Q\left[\left\{v_{k}, v_{k+2}\right\} ; K\right]$ that are all coloured differently. Without loss of generality, $w$ is in $\widehat{v_{k} v_{k+1}}$. Each vertex $x \in T(w)$ is in $\widehat{v_{k} v_{k+1}}$, as otherwise $x w$ crosses $k$ edges in $Q\left[\left\{v_{k}, v_{k+1}\right\} ; K\right]$ that are all coloured differently. Therefore, all nine vertices in $T(w)$ are in $\widehat{v_{k} v_{k+1}}$. By the pigeonhole principle, at least one of $\widehat{v_{k} w}$ or $\widehat{w v_{k+1}}$ contains two vertices from $T_{i}(w)$ and two vertices from $T_{j}(w)$ for some $i, j \in\{2,3,4\}$ with $i \neq j$. Let $x_{1}, x_{2}, x_{3}, x_{4}$ be these four vertices in clockwise order in $\widehat{v_{k} w}$ or $\widehat{w v_{k+1}}$.

Case 1. $x_{1}, x_{2}, x_{3}$ and $x_{4}$ are in $\widehat{v_{k} w}$ : By $(\star)$, the edges in $Q[\{w\} ; K]$ are coloured $2,3, \ldots, k$. Thus $x_{2} v_{k+1}$, which crosses all the edges in $Q[\{w\} ; K]$, is coloured 1. At least one of the vertices in $\left\{x_{2}, x_{3}, x_{4}\right\}$ is adjacent to $\left\{K \backslash\left\{u_{1}, u_{i}\right\}\right\}$ and at least one to $\left\{K \backslash\left\{u_{1}, u_{j}\right\}\right\}$. Thus, by $(\star)$, the edges in $Q\left[\left\{x_{2}, x_{3}, x_{4}\right\} ; K\right]$ are coloured $2,3, \ldots, k$. Thus $x_{1} w$, which crosses all the edges of $Q\left[\left\{x_{2}, x_{3}, x_{4}\right\} ; K\right]$ is coloured 1 . Thus $x_{2} v_{k+1}$ and $x_{1} w$ cross and are both coloured 1 , which is the desired contradiction.

Case 2. $x_{1}, x_{2}, x_{3}$ and $x_{4}$ are in $\widehat{w v_{k+1}}$ : As in Case 1, the edges in $Q\left[\left\{x_{2}, x_{3}, x_{4}\right\} ; K\right]$ are coloured $2,3, \ldots, k$. Thus $x_{1} v_{k+1}$, which crosses all the edges in $Q\left[\left\{x_{2}, x_{3}, x_{4}\right\} ; K\right]$, is coloured 1 . Since the edges in $Q\left[\left\{x_{1}, x_{2}, x_{3}\right\} ; K\right]$ are coloured $2,3, \ldots, k$, the edge $x_{4} w$, which crosses all the edges of 
$Q\left[\left\{x_{1}, x_{2}, x_{3}\right\} ; K\right]$, is coloured 1 . Thus $x_{1} v_{k+1}$ and $x_{4} w$ cross and are both coloured 1 , which is the desired contradiction.

Finally, observe that $|V(Q)|=|K|+|S|+|T|+\sum_{w \in Q}|T(w)|=|K|+11|S|=k+11\left(2 k^{2}+1\right)$. Adding more $k$-simplicial vertices to $Q$ does not reduce its book thickness. Moreover, it is simple to verify that the graph obtained from $Q$ by adding simplicial vertices onto $K$ has a smooth degree-4 tree decomposition of width $k$. Thus for all $n \geq 11\left(2 k^{2}+1\right)+k$, there is a $k$-tree $G$ with $n$ vertices and $\mathrm{bt}(G)=k+1$ that has the desired tree decomposition.

\section{Final Thoughts}

For $k \geq 3$, the minimum book thickness of a $k$-tree is $\left\lceil\frac{k+1}{2}\right\rceil$ (since every $k$-tree contains $K_{k+1}$, and bt $\left(K_{k+1}\right)=\left\lceil\frac{k+1}{2}\right\rceil$; see $($ Bernhart and Kainen, 1979)). However, we now show that the range of book thicknesses of sufficiently large $k$-trees is very limited.

Proposition 1 Every $k$-tree $G$ with at least $\frac{1}{2} k(k+1)$ vertices has book thickness $k-1, k$ or $k+1$.

Proof: Ganley and Heath (2001) proved that bt $(G) \leq k+1$. It remains to prove that bt $(G) \geq k-1$ assuming $|V(G)| \geq \frac{k(k+1)}{2}$. Numerous authors Bernhart and Kainen, 1979 . Cottafava and D'Antona. 1984: Keys, 1975) observed that $|E(G)|<($ bt $(G)+1)|V(G)|$ for every graph $G$. Thus

$$
(k-1)|V(G)| \leq k|V(G)|-\frac{1}{2} k(k+1)=|E(G)|<(\operatorname{bt}(G)+1)|V(G)| .
$$

Hence $k-1<\operatorname{bt}(G)+1$. Since $k$ and bt $(G)$ are integers, bt $(G) \geq k-1$.

We conclude the paper by discussing some natural open problems regarding the computational complexity of calculating the book thickness for various classes of graphs.

Proposition 1 begs the question: Is there a characterisation of the $k$-trees with book thickness $k-1$, $k$ or $k+1$ ? And somewhat more generally, is there a polynomial-time algorithm to determine the book thickness of a given $k$-tree? Note that the $k$-th power of paths are an infinite class of $k$-trees with book thickness $k-1$; see (Swaminathan et al., 1995).

$k$-trees are the edge-maximal chordal graphs with no $(k+2)$-clique, and also are the edge-maximal graphs with treewidth $k$. Is there a polynomial-time algorithm to determine the book thickness of a given chordal graph? Is there a polynomial-time algorithm to determine the book thickness of a given graph with bounded treewidth? 


\section{References}

F. R. Bernhart and P. C. Kainen. The book thickness of a graph. J. Combin. Theory Ser. B, 27(3):320-331, 1979. doi:10.1016/0095-8956(79) 90021-2.

G. A. Cottafava and O. D'Antona. Book-thickness and book-coarseness of graphs. In Proc. 5th International Symp. on Network Theory (Sarajevo), pages 337-340, 1984.

E. Di Giacomo, W. Didimo, G. Liotta, and S. K. Wismath. Book embeddability of series-parallel digraphs. Algorithmica, 45(4):531-547, 2006. doi:10.1007/s00453-005-1185-7.

R. Diestel. Graph theory, volume 173 of Graduate Texts in Mathematics. Springer, 2nd edition, 2000. http://diestel-graph-theory.com/.

V. Dujmović and D. R. Wood. Graph treewidth and geometric thickness parameters. Discrete Comput. Geom., 37(4):641-670, 2007. doi:10.1007/s00454-007-1318-7

V. Dujmović and D. R. Wood. On linear layouts of graphs. Discrete Math. Theor. Comput. Sci., 6(2):339358, 2004. http://www.dmtcs.org/dmtcs-ojs/index.php/dmtcs/article/view/ 193.

J. L. Ganley and L. S. Heath. The pagenumber of $k$-trees is $O(k)$. Discrete Appl. Math., 109(3):215-221, 2001. doi:10.1016/S0166-218X(00)00178-5.

C. D. Keys. Graphs critical for maximal bookthickness. Pi Mu Epsilon J., 6:79-84, 1975.

L. T. Ollmann. On the book thicknesses of various graphs. In F. Hoffman, R. B. Levow, and R. S. D. Thomas, editors, Proc. 4th Southeastern Conference on Combinatorics, Graph Theory and Computing, volume VIII of Congr. Numer., page 459. Utilitas Math., 1973.

S. Rengarajan and C. E. Veni Madhavan. Stack and queue number of 2-trees. In D.-Z. Du and M. Li, editors, Proc. 1st Annual International Conf. on Computing and Combinatorics (COCOON '95), volume 959 of Lecture Notes in Comput. Sci., pages 203-212. Springer, 1995. doi:10 . 1007 / BFb0030834.

R. P. Swaminathan, D. Giriraj, and D. K. Bhatia. The pagenumber of the class of bandwidth- $k$ graphs is $k-1$. Inform. Process. Lett., 55(2):71-74, 1995. doi:10.1016/0020-0190(95) 00079-R.

M. Togasaki and K. Yamazaki. Pagenumber of pathwidth- $k$ graphs and strong pathwidth- $k$ graphs. Discrete Math., 259(1-3):361-368, 2002. doi:10.1016/S0012-365X(02) 00542-3

J. Vandenbussche, D. B. West, and G. Yu. On the pagenumber of k-trees. SIAM J. Discrete Math., 23(3): 1455-1464, 2009. doi:10 .1137/080714208. 